\title{
Rapid detection of active human cytomegalovirus infection in pregnancy using loop-mediated isothermal amplification
}

\author{
XIAOLI WANG ${ }^{*}$, XIAOYAN LI* ${ }^{*}$, SHUHONG HU, HONGMEI QU, \\ YINGHONG ZHANG, HUIJIE NI and XIAOLIANG WANG \\ Department of Obstetrics and Gynecology, Yantai Yuhuangding Hospital of Qingdao University, \\ Yantai, Shandong 264000, P.R. China
}

Received September 26, 2014; Accepted March 5, 2015

DOI: $10.3892 / \mathrm{mmr} .2015 .3572$

\begin{abstract}
Understanding the association between congenital human cytomegalovirus (HCMV) infection and active maternal HCMV infection during pregnancy is important for maternal and neonatal healthcare. In the present study, a loop-mediated isothermal amplification (LAMP) method was established for the detection of CMV DNA from whole blood or amniotic fluid samples, using reverse transcription-quantitative polymerase chain reaction. The results of the present study demonstrated that the CMV LAMP assay detection was specific for CMV DNA, whereas it did not detect viral DNA from herpes simplex type 1 (HSV-1), HSV-2, varicella zoster virus, HSV-6 or HSV-7. Sensitivity determination using serially-diluted CMV glycoprotein B-containing plasmids, demonstrated that $>10$ copies per tube were detectable using the CMV LAMP method. Furthermore, the detection results, using the LAMP method for 336 whole blood samples, demonstrated that at a threshold of $10^{1}-10^{4}$ copies per tube, the sensitivity of this method was $86.96-100 \%$, the specificity was 97.24-100\%, the positive predictive value was $76.92-100 \%$ and the negative predictive value was $99.05-100 \%$. The results for 11 amniotic fluid samples from pregnant women with whole blood CMV-positive and 15 control amniotic fluid samples, indicated that the CMV LAMP assay was sensitive and specific for CMV detection. In conclusion, in the present study, a CMV LAMP method was developed, which was shown to be sensitive, specific and efficient in the detection of HCMV infection. Furthermore, CMV LAMP is capable of detecting active CMV infection in pregnant women. Therefore, the current
\end{abstract}

Correspondence to: Professor Xiaoliang Wang, Department of Obstetrics and Gynecology, Yantai Yuhuangding Hospital of Qingdao University, 20 Yuhuangding East Road, Yantai, Shandong 264000, P.R. China

E-mail: xiaonliangwang@163.com

*Contributed equally

Key words: active human cytomegalovirus infection, pregnancy, loop-mediated isothermal amplification study provides novel insights into diagnostic approaches for active CMV infection in pregnant women.

\section{Introduction}

Congenital infection with human cytomegalovirus (HCMV), which belongs to the herpesviridae group, and may be termed human herpes virus-5, is the most common intrauterine infection $(1,2)$. Studies have demonstrated an association between active CMV infection of the mother and in utero HCMV transmission. The risk of congenital HCMV infection is higher in infants when the mother acquires an initial CMV infection during pregnancy, compared with that in infants when the mother acquires the infection prior to conception (3-7). Therefore, maternal antibodies against HCMV provide protection against congenital infection $(8,9)$. Congenital HCMV infection poses a high risk of causing congenital disorders. Congenital HCMV infection (15-20\%) leads to long-term disability including sensorineural hearing loss, visual impairment, mental retardation and cognitive defects. Furthermore, $4 \%$ of CMV-infected infants do not survive $(3-5,8,10)$.

Understanding the association between congenital infection and active maternal HCMV infection during pregnancy is important for maternal and neonatal healthcare. Therefore, the identification of active HCMV infection during pregnancy is required. However, $>95 \%$ pregnant females with primary $\mathrm{CMV}$ infection are asymptomatic and, therefore, clinical diagnosis is challenging $(11,12)$. Seroconversion may be used to detect HCMV antibodies during pregnancy. However, it is rarely effective, due to the lack of antibody screening prior to conception, which would enable the identification of seronegativity. Routine viral culturing may be sufficiently sensitive for the identification of CMV. However, this method is labor-intensive and subjective, and it may take $>14$ days for the virus to be propagated and identified (13). Due to the limitations of culture-based methods, targeting the viral genome via quantitative polymerase chain reaction (qPCR) has become an important laboratory tool for the diagnosis and treatment of CMV infection. Previous studies have evaluated qPCR for the detection and quantification of CMV in plasma samples $(14,15)$.

A novel nucleic acid amplification method, loop-mediated isothermal amplification (LAMP), has been reported to detect CMV viral genomic DNA (16). This method has been used 
for the rapid diagnosis of a number of infectious diseases, including herpes viruses (17-19), Epstein-Barr virus (20), hepatitis B Virus (21) and CMV (22). LAMP is capable of amplifying specific sequences of DNA under homoeothermic conditions and requires relatively simple and cost-effective equipment, making it amenable for use in hospital laboratories

In the present study, a simple LAMP assay was established for the detection of CMV in peripheral blood samples from pregnant women. This detection method exhibits the potential for use in point-of-care settings for CMV infection screening and follow-up during pregnancy.

\section{Materials and methods}

Clinical specimens and DNA extraction. Whole blood samples from 336 pregnant women, who were registered at Yantai Yuhuangding Hospital of Qingdao University (Yantai, China), were used in the present study. Amniotic fluid samples (11) were obtained from pregnant women exhibiting CMV-positivity, which was identified using reverse transcription qPCR (RT-qPCR) and LAMP assays. Informed consent was obtained from the patients, and the study was permitted by the Human Research Ethics Committee of Yantai Yuhuangding Hospital of Qingdao University. Samples were initially detected using RT-qPCR and then evaluated using a LAMP assay. Total DNA from whole blood samples or amniotic fluid samples was extracted using a QIAamp DNA Mini kit (Qiagen GmbH, Hilden, Germany), according to the manufacturer's instructions. Total extracted DNA was quantified by measurements at $260 \mathrm{~nm}$ optical density (OD) using a spectrophotometer (NanoDrop 2000; Thermo Fisher Scientific, Wilmington, DE, USA). Extracted DNA samples were stored at $-20^{\circ} \mathrm{C}$ prior to use. Viral DNA isolation was performed from stock viruses of herpes simplex virus type 1 (HSV-1), HSV-2, varicella zoster virus (VZV), HSV-6, HSV-7 and CMV (Sinobio, Beijing, China) using the QIAamp DNA Mini kit.

Primer design for LAMP. The primers for LAMP amplification of the CMV glycoprotein B (gB) gene were designed based on CMV sequence data obtained from Genbank (accession number: M60931). Oligonucleotide primers that were used in the present study were designed using Primer Explorer V4 software (Eiken Chemical Co. Ltd., Tokyo, Japan). Designed primer sequences were subjected to BLAST (http://blast.ncbi.nlm.nih. gov/Blast.cgi) in order to exclude the possibility of cross-reactivity with HSV-1, HSV-2, VZV, HSV-6 and HSV-7. CMV specific primers consisted of two outer (F3 and B3) and two inner primers: Forward inner primer (FIP) and backward inner primer (BIP). Inner primers that recognized both forward and reverse strands of the target DNA were connected by a 'TTTT' linker. And additional loop primers [forward loop primer (LF) and backward loop primer (LB)] were used to promote both the amplification efficiency and acceleration of the reaction. Details of the sequence and location of each nucleotide primer in the target DNA sequences are provided in Fig. 1.

Optimization of LAMP conditions. In order to determine the sensitivity of the CMV LAMP method, part of the gB gene containing the target DNA sequence was amplified using the following primers: Forward TGCCCGACGTCACGGTGGTC and reverse: ACCGACTTCAGGGTACTGG, which was cloned into pGEM-T-Easy plasmid (Promega Corporation, Madison, WI, USA). Optimization of LAMP conditions for CMV and sensitivity determination was determined by amplifying $10^{0}-10^{7}$ copies of CMV gB-containing plasmids. The specificity of the LAMP assay was determined using HSV-1, HSV-2, VZV, HSV-6 and HSV-7 DNA samples as negative controls.

Amplifications were optimized using different conditions: Using 20, 25, 30 or $35 \mu \mathrm{l}$ reaction volumes, including 2,5 or $10 \mu \mathrm{l}$ DNA template, 1 or $2 \mu \mathrm{M}$ inner primers (FIP and $\mathrm{BIP}), 0.1,0.3$ or $0.5 \mu \mathrm{M}$ outer primers (F3 and B3) and 0.5 or $1 \mu \mathrm{M}$ loop primers (LF and LB), $0.5,1$ or $2 \mu \mathrm{l}$ Bst DNA polymerase (Large Fragment; New England Biolabs, Inc., Ipswich, MA, USA), $2 \mathrm{x}$ reaction mix ( 0.5 of the total volume), and supplemented distilled and deionized water $\left(\mathrm{ddH}_{2} \mathrm{O}\right)$. Reaction temperatures were screened at 59,62 or $65^{\circ} \mathrm{C}$ and at the following reaction times: 5, 10, 15, 20, 25, 30 and $35 \mathrm{~min}$. A LAMP turbidimeter TERAMECS (LA200; Teramecs, Co. Ltd., Kyoto, Japan) was used to incubate the mixtures and to measure the turbidity following the LAMP reaction. The turbidity cut-off value was set at $>0.1$ mean \pm 3 standard deviation of the turbidity, from the turbidity values of three negative samples. The LAMP products were also subjected to $1.5 \%$ agarose gel electrophoresis in order to validate the experiments. Gels were visualized under an ultraviolet light following ethidium bromide staining.

CMV-specific RT-qPCR assay of whole-blood and amniotic fluid samples. Primers for the CMV RT-qPCR assay were designed according to previously reported sequences (23) and were synthesized by Sangon Biotech (Shanghai, China). Primers were dissolved in $\mathrm{ddH}_{2} \mathrm{O}$, to $100 \mu \mathrm{M}$ and stored at $-20^{\circ} \mathrm{C}$. The RT-qPCR assay was performed using a One-Step PrimeScript RT-PCR kit (Takara Bio, Inc., Otsu, Japan), using LightCycle 2.0 (Roche Diagnostics GmbH, Mannheim, Germany).

Evaluation of LAMP with clinical specimens. In order to evaluate the LAMP assay in whole blood or amniotic fluid specimens from pregnant women, 336 whole blood samples were tested for CMV using RT-qPCR. Whole blood samples (336) and 11 amniotic fluid samples from RT-qPCR-confirmed CMV-positive pregnant women were then subjected to a LAMP assay using the optimized conditions (25 $\mu 1$ reaction volume, including $3 \mu \mathrm{L}$ DNA template, $1 \mu \mathrm{M}$ inner primers, $0.5 \mu \mathrm{M}$ outer primers and $0.5 \mu \mathrm{M}$ loop primers, $1 \mu \mathrm{l}$ Bst DNA polymerase and $\mathrm{ddH}_{2} \mathrm{O}$. The reaction was performed at $62^{\circ} \mathrm{C}$ for $35 \mathrm{~min}$ ). Sensitivity, specificity, positive predictive value and negative predictive value from the LAMP assays were then calculated using standard formulas and the results of RT-qPCR were used as standards.

\section{Results}

Optimized conditions for LAMP assays. In order to optimize the conditions for CMV LAMP detection, LAMP was conducted under different conditions, including different $\mathrm{Mg}^{2+}$ concentrations, different concentrations of loop primers, and different temperatures and durations. The results suggested that LAMP 
A

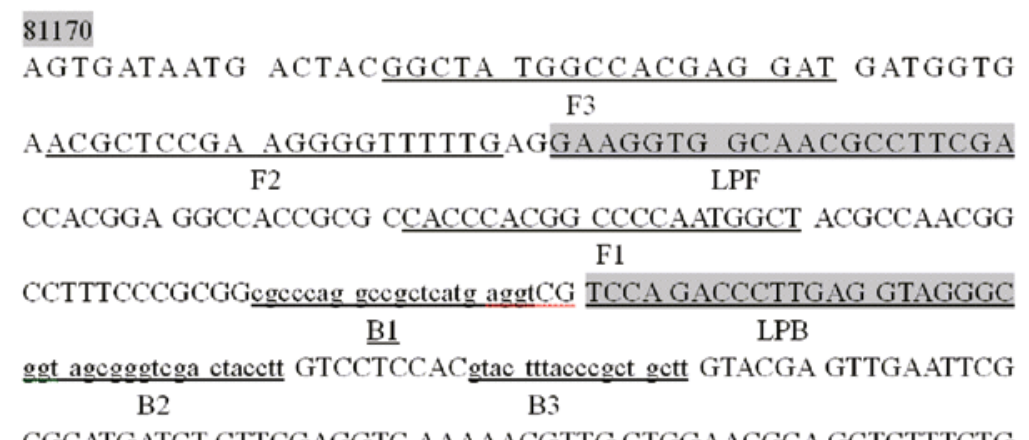

B

\begin{tabular}{ll}
\hline Primers & \multicolumn{1}{c}{ Sequence } \\
\hline F3 & 5' ggctatggccacgaggat 3' \\
B3 & 5' aagcagcgggtaaagtac 3' \\
FIB & 5' agccattggggccgtgggtg TTTT acgctccgaaggggtttttg 3' \\
BIP & 5' cgeccaggcegctcatgaggt TTTT aaggtagtcgaccegctacc 3' \\
LF & 5' gaaggtggcaacgccttcg 3' \\
LB & 5' gccetacctcaagggtctgga 3' \\
\hline
\end{tabular}

Figure 1. Locations and target sequences of the CMV gB gene and the primers for CMV LAMP. (A) Target sequences in the CMV gB gene. (B) Primer sequences for the CMV LAMP. F3, labeled sequence in the target sequence; B3, reverse complementary sequence in the target sequence; FIB, forward internal primer, reverse complementary sequence of F1 + TTTT + labeled F2 sequence; BIP, backwarrd internal primer, labeled B1 sequence + TTTT + reverse complementary sequence of B2; LF, forward loop primer, labeled LPF sequence in the target sequence; LB, backward loop primer, reverse complementary sequence of labeled LPB sequence; CMV, cytomegalovirus; LAMP, loop-mediated isothermal amplification; glycoprotein B, gB.

A

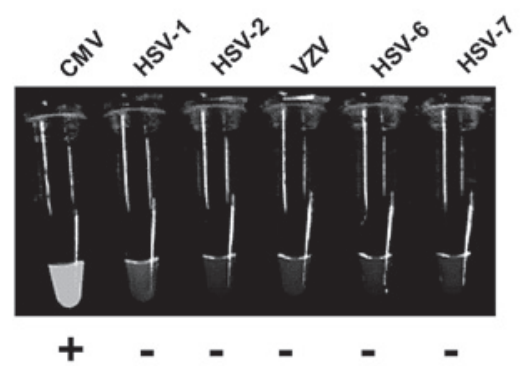

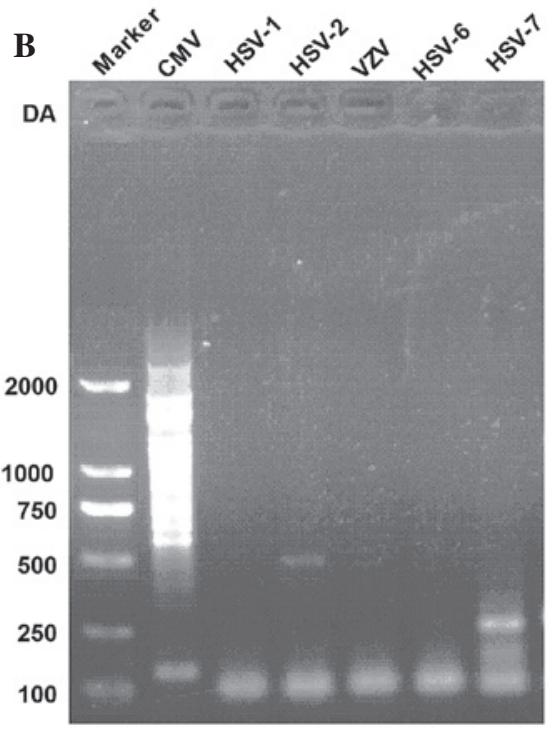

Figure 2. LAMP assay specificity to CMV, targeted to the CMV gB gene. (A) Visual inspection of LAMP assay for CMV, HSV-1, HSV-2, VZV, HSV-6 and HSV-7. (B) Electrophoretic analysis of LAMP product from samples of CMV, HSV-1, HSV-2, VZV, HSV-6 and HSV-7. LAMP, loop-mediated isothermal amplification; CMV, cytomegalovirus; HSV, herpes simplex virus; VSV, varicella zoster virus.

conditions were optimized at a $25-\mu 1$ reaction volume. Final reaction mixtures consisted of $1.6 \mu \mathrm{M}$ inner primers (FIP and BIP), $0.2 \mu \mathrm{M}$ outer primers (F3 and B3), $0.8 \mu \mathrm{M}$ loop primers (LF and LR), $10 \mathrm{mM} \mathrm{MgSO}_{4}, 1 \mu \mathrm{l}$ Bst DNA polymerase and $5 \mu \mathrm{l}$ DNA template. Amplifications were performed at $64^{\circ} \mathrm{C}$ for $30 \mathrm{~min}$ and reactions were terminated at $85^{\circ} \mathrm{C}$ for $5 \mathrm{~min}$.
Specificity of LAMP using turbidity assays and gel electrophoresis. In the present study, the capability of the CMV LAMP assay for the discrimination of CMV from other members of herpesviridae, such as HSV-1, HSV-2, VZV, HSV-6 and HSV-7 was assessed. The results of the present study suggested that CMV exhibits bright turbidity following 
Table I. Performance of the LAMP assay for active CMV infection in whole blood samples.

\begin{tabular}{lccccccc}
\hline & \multicolumn{7}{c}{ LAMP (+/-) } \\
\cline { 3 - 5 } Copies/tube & Total number & RT-qPCR+ & RT-qPCR- & Sensitivity (\%) & Specificity (\%) & PPV (\%) & NPV (\%) \\
\hline$>10^{1}$ & 336 & $20 / 3$ & $0 / 313$ & 86.96 & 100 & 100 & 99.05 \\
$>10^{2}$ & 336 & $21 / 2$ & $1 / 312$ & 91.30 & 99.68 & 94.45 & 99.36 \\
$>10^{3}$ & 336 & $13 / 0$ & $2 / 318$ & 100 & 98.45 & 86.67 & 100 \\
$>10^{4}$ & 336 & $10 / 0$ & $3 / 317$ & 100 & 97.24 & 76.92 & 100
\end{tabular}

PPV, positive predictive value; NPV, negative predictive value; LAMP, loop-mediated isothermal amplification; CMV, cytomegalovirus; RT-qPCR, reverse transcription-quantitative polymerase chain reaction.

Table II. Diagnostic performance of LAMP assay for active CMV infection in amniotic fluid samples.

\begin{tabular}{lcccc}
\hline $\begin{array}{l}\text { Copies per } \\
\text { tube }\end{array}$ & $\begin{array}{c}\text { CMV+ } \\
\text { number }\end{array}$ & Positive & $\begin{array}{c}\text { CMV- } \\
\text { number }\end{array}$ & Negative \\
\hline$>10^{1}$ & 11 & 10 & 15 & 15 \\
$>10^{2}$ & 11 & 11 & 15 & 15 \\
$>10^{3}$ & 11 & 11 & 15 & 15 \\
$>10^{4}$ & 11 & 11 & 15 & 14 \\
\hline
\end{tabular}

Positive total per tube $=11$ and negative total per tube $=15 ;$ LAMP, loop-mediated isothermal amplification; CMV, cytomegalovirus.

a LAMP assay, whereas other members of herpesviridae were not detected using LAMP (Fig. 2A). LAMP products were then analyzed using gel electrophoresis and the results suggested that CMV was successfully amplified, while other viral DNA was not amplified (Fig. 2B). Therefore, primer sets developed in the present study exhibit specificities for the target CMV sequences.

Sensitivity of LAMP using turbidity measurement and $R T-q P C R$. LAMP assay sensitivity was analyzed using the serially-diluted CMV gB-containing plasmid. Serial dilutions of recombinant $\mathrm{pGEM}-\mathrm{T}$ Easy plasmid ranging from $10^{7}-10^{0}$ copies per tube were used in order to determine the detection limits of CMV LAMP. Results demonstrated that the sensitivity of the CMV LAMP assay was 10 copies per tube, according to a real-time turbidimeter at $650 \mathrm{~nm}$ OD (Fig. 3A and B). Reactions were repeated three times. Serially-diluted plasmids were examined, ranging from $10^{6}-10^{-1}$ copies per tube, using RT-qPCR (Fig. 3C). The threshold of RT-qPCR was $10^{-1}$ copies per tube, which was 10 times more sensitive than that of the CMV LAMP assay.

CMV LAMP assay of whole blood specimens from pregnant women (Tables I and II). In order to further evaluate the performance of the CMV LAMP assay for CMV infection detection in pregnant women, CMV LAMP assays were conducted in 336 whole blood samples and 11 amniotic fluid samples from pregnant women. Samples were tested using RT-qPCR. Positive samples $(10,13,20$ or 21$)$, with a threshold of 10 copies, were
A

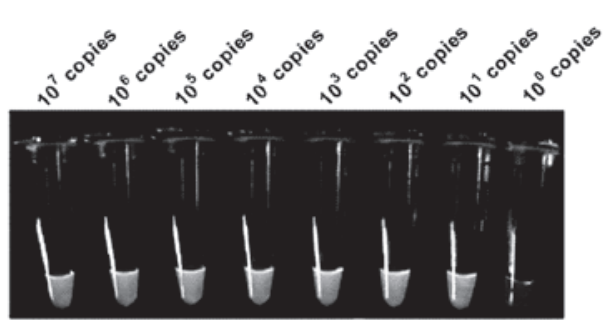

B

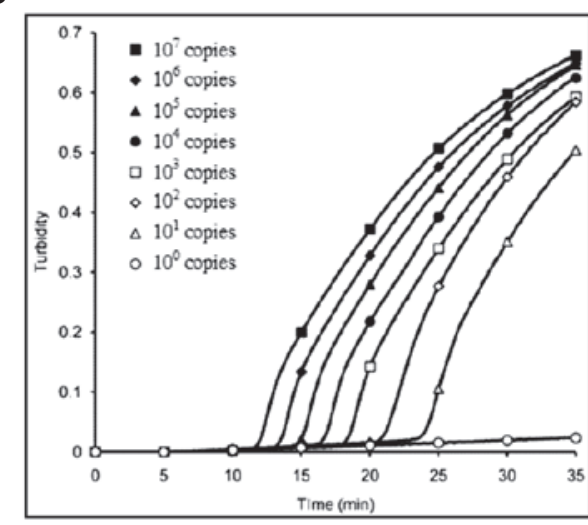

C

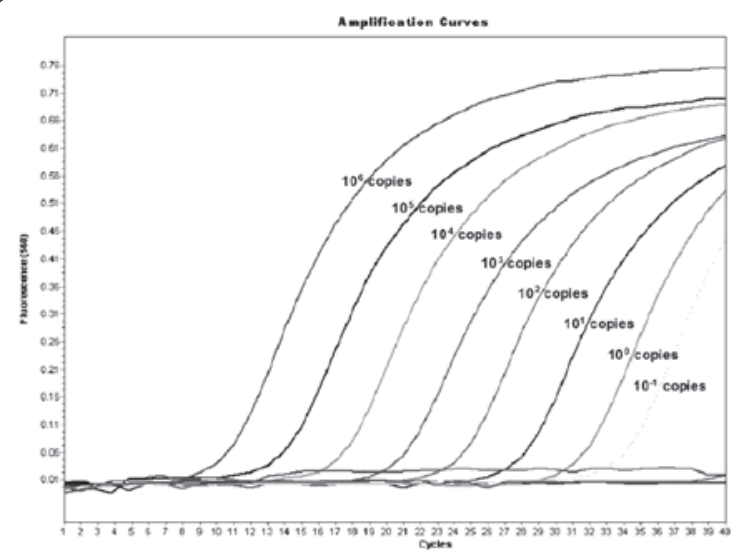

Figure 3. Sensitivity of the CMV LAMP assay. (A) Visual inspection of LAMP assay results for serially diluted $\left(10^{0}-10^{7}\right.$ copies per tube) CMV gB-containing plasmids. (B) Turbidity results of the LAMP products for the serially diluted CMV gB-containing plasmids. (C) Reverse transcription quantitative-polymerase chain reaction results for the serially diluted $\left(10^{-1}-10^{6}\right.$ copies per tube) $\mathrm{CMV}$ gB-containing plasmids. CMV, cytomegalovirus; $\mathrm{gB}$, glycoprotein $\mathrm{B}$; LAMP, loop-mediated isothermal amplification. 
confirmed using RT-qPCR. RT-qPCR-positive samples, 10 and 13, were confirmed as positive according to the CMV LAMP, with a threshold of $10^{3}$ or $10^{4}$ copies per tube, respectively and $100 \%$ sensitivity. However, samples 2 and 3, which were negative according to RT-qPCR, were shown to be positive according to the CMV LAMP assay, with a specificity of 98.45 and $97.24 \%$, PPV values of 86.67 and $76.92 \%$, respectively; NPV for both thresholds were $100 \%$ (Table I). The following values were observed at $10^{1}$ and $10^{2}$ copies per tube compared with those at $10^{3}$ and $10^{4}$ : Sensitivity decreased to 86.96 and $91.30 \%$, specificity increased to 100 and $99.68 \%$, PPV increased to 100 and $94.45 \%$, and NPV decreased to 99.05 or $99.36 \%$, at $10^{1}$ and $10^{2}$ copies per tube respectively. In order to reconfirm the sensitivity and specificity of the CMV LAMP assay, 11 amniotic fluid samples were examined from pregnant women with whole blood CMV-positive and 15 control amniotic fluid samples were examined, from pregnant women without active CMV infection. The results indicated that 11 samples were positive, with a threshold of $10^{2}, 10^{3}$ or $10^{4}$, while the 15 control samples were negative, with a threshold of $10^{1}, 10^{2}$ or $10^{3}$ (Table II). Overall, the CMV LAMP method performed well in the detection of CMV infection.

\section{Discussion}

LAMP reaction requires DNA polymerase with strand displacement activity and $>4$ specifically designed primers. During the first step, a stem-loop DNA structure is constructed, in which the sequences of both DNA ends are derived from the inner primers. Subsequently, one inner primer hybridizes to the loop on the LAMP cycle product and initiates strand displacement DNA synthesis, yielding the original stem-loop and new stem-loop DNA with a stem that is twice as long. The final products are termed stem-loop DNAs, and have several inverted repeats of the target DNA and cauliflower-like structures with multiple loops, amplifying $<10^{9}$ copies of the target. LAMP is a rapid and simple technique for the amplification of specific DNA sequences that has advantages over PCR $(24,25)$. The most significant advantage of LAMP is its ability to amplify specific sequences of DNA at a constant temperature $\left(63-65^{\circ} \mathrm{C}\right)$, without thermocycling. In addition, $<45 \mathrm{~min}$ are required in order to amplify the target sequences. Given these advantages, LAMP may be adopted for widespread use in hospital laboratories.

In the present study a CMV LAMP assay was established for the detection of CMV DNA in pregnant women with CMV infection, using RT-qPCR in order to confirm active CMV infection. Following optimization of the PCR protocol, the components for CMV LAMP included a $25-\mu 1$ reaction volume with $1.6 \mu \mathrm{M}$ inner primers, $0.2 \mu \mathrm{M}$ outer primers, $0.8 \mu \mathrm{M}$ loop primers, $10 \mathrm{mM} \mathrm{MgSO}_{4}, 1 \mu \mathrm{l}$ Bst DNA polymerase and $5 \mu \mathrm{l}$ DNA template. The amplification was conducted at $64^{\circ} \mathrm{C}$ for $30 \mathrm{~min}$. This PCR method was specific for the amplification of CMV DNA and it did not amplify HSV-1, HSV-2, VZV, HSV-6 or HSV-7, which belong to the same herpesviridae family. Sensitivity determination using the serially-diluted CMV gB-containing plasmids demonstrated that $>10$ copies per tube were detectable using the CMV LAMP method, which had a 10 fold lower sensitivity level compared with that of RT-qPCR.
Furthermore, the CMV LAMP assay performed well in the detection of CMV infection. The detection results for 336 whole blood samples demonstrated that, at a threshold of $10^{1}-10^{4}$ copies per tube, the sensitivity of the LAMP assay for the detection of CMV infection was 86.96-100\%, specificity was $97.24-100 \%$, PPV was $76.92-100 \%$ and NPV was 99.05-100\%. The LAMP assay was sensitive and specific for the detection of CMV in 11 amniotic fluid samples from CMV-positive pregnant women and in 15 control amniotic fluid samples. Overall, the CMV LAMP method performed well in the detection of CMV infection.

In conclusion, a CMV LAMP method was developed, which was highly sensitive, specific, simple and timesaving. Furthermore, it performed well in the detection of active CMV infection in pregnant women. Therefore, the present study provides novel insights into the detection of active CMV infection in pregnant women.

\section{Acknowledgements}

The present study was supported by a grant from Yantai Yuhuangding Hospital of Qingdao University.

\section{References}

1. Adler SP and Marshall B: Cytomegalovirus infections. Pediatr Rev 28: 92-100, 2007.

2. Enders G, Daiminger A, Bäder U, Exler S and Enders M: Intrauterine transmission and clinical outcome of 248 pregnancies with primary cytomegalovirus infection in relation to gestational age. J Clin Virol 52: 244-246, 2011

3. Coll O, Benoist G, Ville Y, Weisman LE, Botet F, Anceschi MM, Greenough A, Gibbs RS and Carbonell-Estrany X; WAPM Perinatal Infections Working Group: Guidelines on CMV congenital infection. J Perinat Med 37: 433-445, 2009.

4. Revello MG, Fabbri E, Furione M, Zavattoni M, Lilleri D, Tassis B, Quarenghi A, Cena C, Arossa A, Montanari L, et al: Role of prenatal diagnosis and counseling in the management of 735 pregnancies complicated by primary human cytomegalovirus infection: A 20-year experience. J Clin Virol 50: 303-307, 2011.

5. Guerra B, Simonazzi G, Banfi A,Lazzarotto T, Farina A,Lanari M and Rizzo N: Impact of diagnostic and confirmatory tests and prenatal counseling on the rate of pregnancy termination among women with positive cytomegalovirus immunoglobulin $\mathrm{M}$ antibody titers. Am J Obstet Gynecol 196: e1-e6, 2007.

6. Fowler KB, Stagno S, Pass RF, Britt WJ, Boll TJ and Alford CA: The outcome of congenital cytomegalovirus infection in relation to maternal antibody status. N Engl J Med 326: 663-667, 1992.

7. Stagno S, Pass RF, Cloud G, Britt WJ, Henderson RE, Walton PD, Veren DA, Page F and Alford CA: Primary cytomegalovirus infection in pregnancy. Incidence, transmission to fetus, and clinical outcome. JAMA 256: 1904-1908, 1986.

8. Manicklal S, Emery VC, Lazzarotto T, Boppana SB and Gupta RK: The 'silent' global burden of congenital cytomegalovirus. Clin Microbiol Rev 26: 86-102, 2013.

9. Revello MG and Gerna G: Pathogenesis and prenatal diagnosis of human cytomegalovirus infection. J Clin Virol 29: 71-83, 2004.

10. Kenneson A and Cannon MJ: Review and meta-analysis of the epidemiology of congenital cytomegalovirus (CMV) infection. Rev Med Virol 17: 253-276, 2007.

11. Revello MG and Gerna G: Diagnosis and management of human cytomegalovirus infection in the mother, fetus, and newborn infant. Clin Microbiol Rev 15: 680-715, 2002.

12. Lazzarotto T, Guerra B, Lanari M, Gabrielli L and Landini MP: New advances in the diagnosis of congenital cytomegalovirus infection. J Clin Virol 41: 192-197, 2008.

13. de Vries JJ, van der Eijk AA, Wolthers KC, Rusman LG, Pas SD, Molenkamp R, Claas EC, Kroes AC and Vossen AC: Real-time PCR versus viral culture on urine as a gold standard in the diagnosis of congenital cytomegalovirus infection. J Clin Virol 53: 167-170, 2012. 
14. Boaretti M, Sorrentino A, Zantedeschi C, et al: Quantification of cytomegalovirus DNA by a fully automated real-time PCR for early diagnosis and monitoring of active viral infection in solid organ transplant recipients. J Clin Virol 56: 124-128, 2013.

15. Bravo D, Clari MA, Costa E, Muñoz-Cobo B, Solano C, José Remigia M and Navarro D: Comparative evaluation of three automated systems for DNA extraction in conjunction with three commercially available real-time PCR assays for quantitation of plasma cytomegalovirus DNAemia in allogeneic stem cell transplant recipients. J Clin Microbiol 49: 2899-2904, 2011.

16. Notomi T, Okayama H, Masubuchi H, Yonekawa T, Watanabe K, Amino $\mathrm{N}$ and Hase T: Loop-mediated isothermal amplification of DNA. Nucleic Acids Res 28: E63, 2000.

17. Enosawa M, Kageyama S, Sawai K, et al: Use of loop-mediated isothermal amplification of the IS900 sequence for rapid detection of cultured Mycobacterium avium subsp. paratuberculosis. J Clin Microbiol 41: 4359-4365, 2003.

18. Iwamoto T, Sonobe T and Hayashi K: Loop-mediated isothermal amplification for direct detection of Mycobacterium tuberculosis complex, $M$. avium, and $M$. intracellulare in sputum samples. J Clin Microbiol 41: 2616-2622, 2003.

19. Hagiwara M, Sasaki H, Matsuo K, Honda M, Kawase M and Nakagawa H: Loop-mediated isothermal amplification method for detection of human papillomavirus type $6,11,16$, and 18 . J Med Virol 79: 605-615, 2007.
20. Iwata S, Shibata Y, Kawada J, Hara S, Nishiyama Y, Morishima T, Ihira M, Yoshikawa T, Asano Y and Kimura H: Rapid detection of Epstein-Barr virus DNA by loop-mediated isothermal amplification method. J Clin Virol 37: 128-133, 2006.

21. Nyan DC, Ulitzky LE, Cehan N, Williamson P, Winkelman V, Rios M and Taylor DR: Rapid detection of hepatitis B virus in blood plasma by a specific and sensitive loop-mediated isothermal amplification assay. Clin Infect Dis 59: 16-23, 2014.

22. Suzuki R, Yoshikawa T, Ihira M, Enomoto Y, Inagaki S, Matsumoto K, Kato K, Kudo K, Kojima S and Asano Y: Development of the loop-mediated isothermal amplification method for rapid detection of cytomegalovirus DNA. J Virol Methods 132: 216-221, 2006.

23. Nefzi F, Ben SN, Khelif A, Feki S, Aouni $M$ and Gautheret-Dejean A: Quantitative analysis of human herpesvirus- 6 and human cytomegalovirus in blood and saliva from patients with acute leukemia. J Med Virol 87: 451-460, 2015.

24. Notomi T, Okayama H, Masubuchi H, Yonekawa T, Watanabe K, Amino $\mathrm{N}$ and Hase T: Loop-mediated isothermal amplification of DNA. Nucleic Acids Res 28: E63, 2000.

25. Nagamine K, Hase T and Notomi T: Accelerated reaction by loop-mediated isothermal amplification using loop primers. Mol Cell Probes 16: 223-229, 2002. 